\title{
Thermoresponsive Chemical Connectors Based on Hybrid Nanowire Forests**
}

\author{
Hyunhyub Ko, Zhenxing Zhang, Yu-Lun Chueh, Eduardo Saiz, and Ali Javey*
}

Micro- and nanostructured surfaces found in biological systems have inspired the fabrication of functional materials with unique optical, chemical, and mechanical properties. ${ }^{[1]}$ Inspired by the nanofibrillar structures of gecko adhesives, ${ }^{[2]}$ we recently reported self-selective, chemical connectors (i.e., fasteners) based on interpenetrating nanowire (NW) forests, which primarily use the highly tunable van der Waals (vdW) interactions to enable efficient binding of components at both macro- and microscales. ${ }^{[3]}$ Of particular interest to certain practical applications are programmable fasteners that can change their adhesion properties on command, for example, in response to external stimuli. In this regard, herein, we report programmable NW fasteners that reversibly change their wet adhesion strength in response to a thermal change of the environment. The thermoresponsive NW fasteners are based on core/multishell hybrid NW forests with an outer shell of poly( $N$-isopropylacrylamide) (PNIPAM). PNIPAM is a thermoresponsive hydrogel with a lower critical solution temperature (LCST) of approximately $32^{\circ} \mathrm{C}$ in water. ${ }^{[4]}$ Specifically, at room temperature, PNIPAM absorbs water, resulting in the swelling of the polymer and hydrophilic surface properties. However, PNIPAM shrinks at temperatures higher than the LCST and transforms to a hydrophobic state. We utilized this well known property of PNIPAM in conjunction with high aspect ratio nanofibrillar structures to enable programmable fasteners with tunable properties.

[*] Dr. H. Ko, Z. Zhang, Dr. Y.-L. Chueh, Prof. A. Javey Department of Electrical Engineering and Computer Sciences and

Berkeley Sensor and Actuator Center

University of California at Berkeley, Berkeley, CA 94720 (USA)

E-mail: ajavey@eecs.berkeley.edu

and

Materials Sciences Division, Lawrence Berkeley National Laboratory Berkeley, CA 94720 (USA)

Fax: (+1) 510-643-7846

Z. Zhang

Key Laboratory for Magnetism and Magnetic Materials of the Ministry of Education, Lanzhou University (China)

Dr. E. Saiz

Materials Sciences Division, Lawrence Berkeley National Laboratory (USA)

[***] This work was supported by DARPA/DSO, NSF Center of Integrated Nanomechanical Systems, and Berkeley Sensor and Actuator Center. Z.Z. acknowledges a fellowship from the China Scholarship Council. The nanowire synthesis part of this project was supported by a Laboratory Directed Research and Development grant from Lawrence Berkeley National Laboratory.

Dupporting information for this article is available on the WWW under http://dx.doi.org/10.1002/anie.200904724.
The fabrication procedure for the thermoresponsive NW fasteners is outlined in Figure $1 \mathrm{a}$. First, Ge/parylene core/ shell NW forests were prepared by growing Ge NW forests
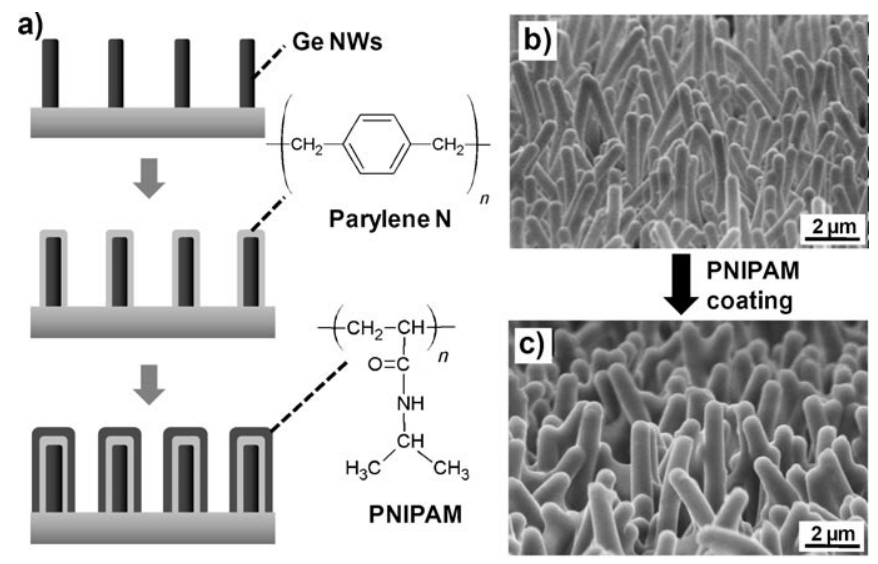

Figure 1. a) Fabrication procedure for a thermoresponsive NW forest, used as one mate of the proposed unisex fasteners. b,c) Scanning electron microscopy (SEM) images of Ge/parylene NW forests before and after the deposition of the PNIPAM outer shell.

(length ca. $10 \mu \mathrm{m}$, diameter $20-30 \mathrm{~nm}$ ) on $\mathrm{Si} / \mathrm{SiO}_{2}$ substrates and then depositing a parylene $\mathrm{N}$ shell (ca. $200 \mathrm{~nm}$ ) as we previously reported elsewhere. ${ }^{[3]}$ Next, a thin layer of PNIPAM shell was deposited on the NW arrays by photopolymerization of a solution mixture of NIPAM monomers, 2,2'-diethoxyacetophenone photoinitiator, and $N, N$-methylenebisacrylamide cross-linker (see the Experimental Section for process details). In this structure, the Ge core with high Young's modulus serves as the backbone template. The parylene shell provides surface compliance and structural robustness, without which the inorganic NWs readily break and detach from the substrate. Furthermore, the hydrophobic parylene shell, which enhances the stiffness of the NWs, minimizes the aggregation of the NW arrays during the PNIPAM deposition processing. Finally, the PNIPAM outer shell serves as the thermoresponsive layer for modulation of the adhesion properties.

The hybrid NW forests modified with thermoresponsive PNIPAM outer shell exhibited thermally tunable surface wetting properties. Specifically, a transition of the water contact angle (CA) from 70 to $138^{\circ}$ is observed as the temperature is raised to greater than $32^{\circ} \mathrm{C}$ (Figure 2). In contrast, a planar PNIPAM film shows a change in water CA from 60 to $76^{\circ}$ as the temperature is raised above LCST (Figure 2), which is consistent with previously reported 
a)
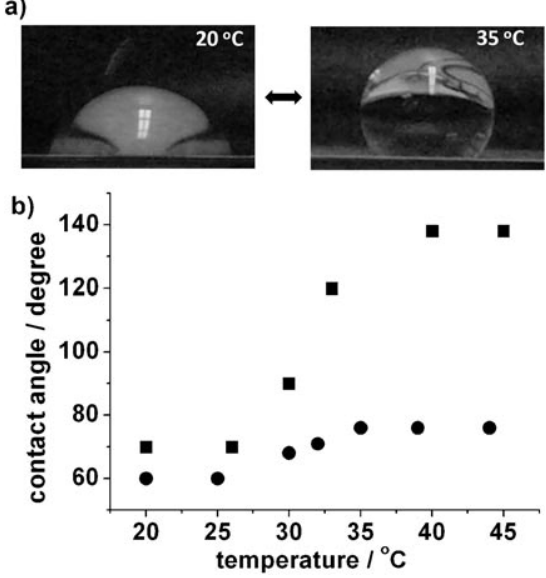

Figure 2. Thermoresponsive wetting properties of NW forests. a) Water droplet on the surface of Ge/parylene/PNIPAM core/multishell NW forests at 20 (left) and $35^{\circ} \mathrm{C}$ (right). b) Contact angle as a function of temperature for Ge/payrlene/PNIPAM NW forests (-) and planar PNIPAM films $(\bullet)$.

studies. ${ }^{[4,5]}$ The significant enhancement of the surface wetting modulation for the NW forests compared with the planar surfaces results from the geometric amplification effects of the wetting properties. The hydrophobicity of a rough surface has been known to increase with the increase of water/air interface area. ${ }^{[6]}$ In our study, the micro- and nanoscale roughness of NW forests can trap air below the water drop and thus intensify the water contact angle relative to that of the planar film. This change of the contact angle of hybrid NW forests confirms the phase transition (i.e., hydrophilic/ hydrophobic, swelling/shrinking) of PNIPAM at the LCST $\left(\right.$ ca. $32^{\circ} \mathrm{C}$ ), which in turn affects the shear adhesion of NW fasteners as described below.

Shear adhesion strengths were measured in water (Figure 3 a schematic) as a function of temperature to investigate the thermoresponsive wet adhesion properties of the NW fasteners. The fasteners showed minimal shear adhesion strength of around $0.2 \mathrm{~N} \mathrm{~cm}^{-2}$ at temperatures of $20-30^{\circ} \mathrm{C}$ (Figure $3 \mathrm{a}$ ). However, a drastic enhancement of the shear adhesion strength by 170 times $\left(34 \mathrm{~N} \mathrm{~cm}^{-2}\right)$ was observed when the water temperature was increased to $32^{\circ} \mathrm{C}$. The sharp transition of shear adhesion strength at $32^{\circ} \mathrm{C}$ arises from the phase transition of PNIPAM at the LCST, directly affecting the interactions between PNIPAM surfaces. Our previous studies of Ge/parylene NW fasteners in both wet and dry environments have shed light on the dominant role of vdW interactions in the adhesion properties. ${ }^{[7]}$ Since the vdW interactions between two identical surfaces with dielectric constant of $\varepsilon_{1}$ in a liquid medium with dielectric constant of $\varepsilon_{2}$ is known to depend on $F=\left[\left(\varepsilon_{1}-\varepsilon_{2}\right) /\left(\varepsilon_{1}+\varepsilon_{2}\right)\right]^{2}{ }^{[8]}$ the adhesion can be readily tuned by changing the dielectric constant of the surfaces (in this case, PNIPAM outer shell).

Previous studies have shown that the dielectric constant of PNIPAM decreases from around 63 to around 17 when the temperature is increased from $15^{\circ} \mathrm{C}$ to $40^{\circ} \mathrm{C} \cdot{ }^{[9]} \mathrm{A}$ similar temperature dependency of the dielectric constant is expected for the PNIPAM outer shell of our NW fasteners, which
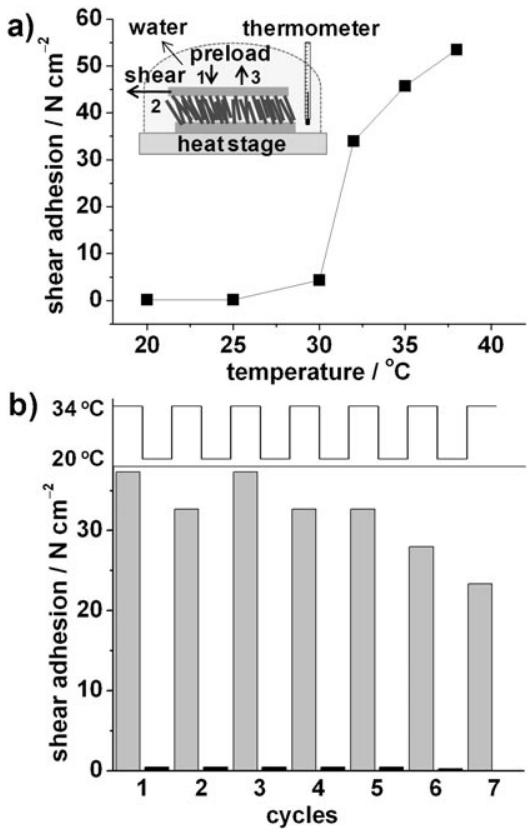

Figure 3. Thermoresponsive shear adhesion strength of hybrid NW fasteners. a) Shear wet adhesion strengths of the thermoresponsive NW fasteners involving the engagement of two NW forest substrates. The forests consist of $\mathrm{Ge} /$ parylene/PNIPAM core/multishell NWs. The inset shows the adhesion tests by sequential procedures of: 1) applying a normal preload stress (ca. $70 \mathrm{~N} \mathrm{~cm}^{-2}$ ) to engage the fasteners, 2) applying a shear force, and 3) removing the preload stress. b) Reversible modulation of shear adhesion strength of the thermoresponsive NW fasteners at two different water temperatures $\left(20\right.$ and $\left.34^{\circ} \mathrm{C}\right)$. The gray and black bars correspond to the adhesion strength at 34 and $20^{\circ} \mathrm{C}$, respectively. The samples were allowed to equilibrate for $20 \mathrm{~min}$ at each temperature prior to performing the adhesion tests.

results in large modulation of $\mathrm{vdW}$ interactions $\left[F\left(40^{\circ} \mathrm{C}\right) /\right.$ $\left.F\left(15^{\circ} \mathrm{C}\right) \approx 30\right]$. In fact, this thermoresponsive modulation of binding interactions has been previously utilized to induce flocculation of PNIPAM microgel particles dispersed in water, ${ }^{[10]}$ and to control the adsorption and release of proteins on PNIPAM surfaces. ${ }^{[5]}$ In addition to the modulation of $\varepsilon_{1}$, hydrophobic effects arising from the thermally tunable surface wetting properties may contribute to the observed thermoresponsive adhesion behavior. ${ }^{[4]}$ Specifically, the drastic transition to a superhydrophoic state at temperatures higher than the LCST (Figure 2) enhances the NW binding interactions in water. Finally, the induced swelling/deswelling behavior of PNIPAM may affect the filling factor and thus the interpenetration efficiency and the contact area of the NW arrays. However, the change in the filling factor is negligible with the overall NW diameter change of only $6-10 \%$ (corresponding to $25-40 \%$ change in the thickness of the PNIPAM outer shell) between the wet and dry states as estimated from the environmental SEM analyses (Figure S1 in the Supporting Information). Most likely, the observed thermoresponsive adhesion trend arises from a complex combination of the forces described above.

Notably, the thermoresponsive NW fasteners show selective adhesion behavior with low binding strengths to non-self- 
similar surfaces. As can be seen in Figure S2 (in the Supporting Information), PNIPAM coated NW forests show minimal adhesion $\left(<1.6 \mathrm{~N} \mathrm{~cm}^{-2}\right)$ on flat glass surfaces for the temperature range $20-40^{\circ} \mathrm{C}$. Furthermore, the adhesion strength between PNIPAM coated NW forests and planar PNIPAM films is only approximately $4 \mathrm{Ncm}^{-2}$ at high temperatures (Figure S2), which is 11 times lower than when NW forests are engaged with self-similar surfaces (i.e., in a fastener configuration). This selective binding is due to the drastic amplification of the contact area for NW/NW interfaces relative to the $\mathrm{NW} /$ planar surfaces for the relatively stiff NWs explored in this study. ${ }^{[3,7]}$ Selective binding is an important characteristic of a fastener technology, as distinct from global adhesives, such as synthetic gecko materials, which are specifically designed to bind nonselectively to most surfaces.

Reversible adhesion with the capability of strong binding and easy detachment, controllable by temperature, is an important property of NW fasteners for practical applications. To demonstrate the reversible switching of adhesion strength of NW fasteners, we repeatedly measured the shear adhesion of NW fasteners by alternately transferring them between high $\left(34^{\circ} \mathrm{C}\right.$, above LCST) and low $\left(20^{\circ} \mathrm{C}\right.$, below LCST) temperature water baths. For each test, we immersed the NW forests in water for $20 \mathrm{~min}$ to provide enough time for swelling or shrinking of PNIPAM outer shells before engaging the NW fasteners for adhesion tests. The thermoresponsive wet adhesion behavior of the NW fasteners is reversible. Figure $3 \mathrm{~b}$ shows the reversible switching of the shear adhesion strength of a fastener with strong adhesion $\left(23-37 \mathrm{~N} \mathrm{~cm}^{-2}\right)$ at $34^{\circ} \mathrm{C}$ (gray bars in Figure $3 \mathrm{~b}$ ) and minimal adhesion $\left(<0.5 \mathrm{~N} \mathrm{~cm}^{-2}\right)$ at $20^{\circ} \mathrm{C}$ (red bars in Figure $\left.3 \mathrm{~b}\right)$ for seven cycles of operation. The thermoresponsive fasteners show excellent reversible modulation of adhesion strengths over multiple cycles, exhibiting only minimal degradation, as depicted in Figure $3 \mathrm{~b}$. The slight degradation of the shear adhesion of NW fasteners is due to surface damage of the NW fasteners during the adhesion test.

Since the adhesion strength of NW fasteners depends on the level of phase transition of PNIPAM shell above LCST, it is important to test the time required for NW fasteners to exhibit their maximum adhesion strength. To further elucidate the phase transition properties of PNIPAM shell and operational mechanics of NW fasteners, we examined the response time of NW fasteners by measuring the shear adhesion strength of NW fasteners at specified time intervals after immersing the samples in high temperature (above LCST) water bath. We observed a drastic linear enhancement in the shear adhesion within the first $5 \mathrm{~min}$ after transferring the fastener samples from a low $\left(20^{\circ} \mathrm{C}\right)$ to high $\left(35^{\circ} \mathrm{C}\right)$ temperature water bath (Figure 4). Beyond the first $5 \mathrm{~min}$, the adhesion remained fairly constant at $40-45 \mathrm{~N} \mathrm{~cm}^{-2}$. This result indicates that NW fasteners are fully operational within $5 \mathrm{~min}$, beyond which the adhesion strength is close to the maximum value. Notably, the response times of the NW fasteners are significantly faster than the typical deswelling rate of millimeter-scale, bulk PNIPAM films (few minutes vs. several hours). ${ }^{[11,12]}$ The faster response time is attributed to the use of nanofibrillar structures with large surface areas and

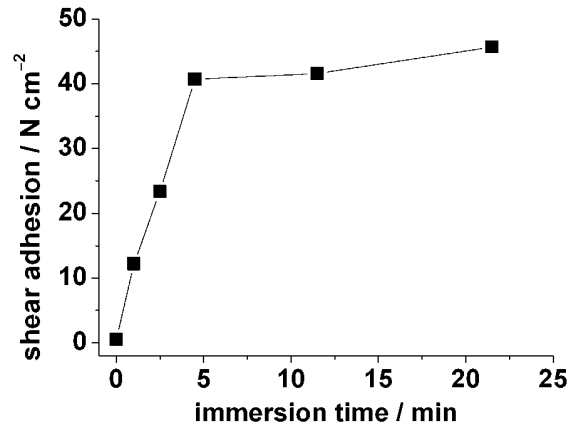

Figure 4. Shear adhesion strength as a function of the immersion time in water when the thermoresponsive NW fasteners were transferred from a low $\left(20^{\circ} \mathrm{C}\right)$ to high $\left(35^{\circ} \mathrm{C}\right)$ temperature water bath.

relatively thin PNIPAM films (ca. $100 \mathrm{~nm}$ thick shells) in this study, requiring minimal water diffusion lengths.

In summary, a unique platform for programmable fasteners based on core/multishell NW forests that reversibly change their wet adhesion strength by around 170 times in response to a water temperature change of less than $5^{\circ} \mathrm{C}$ is reported. The unique properties of the thermoresponsive PNIPAM outer shell in conjunction with the 3D geometric configuration of the high aspect NWs enables highly unique binding properties that arise primarily from tunable vdW interactions along with hydrophobic surface effects. In the future, programmable fasteners that respond to other external stimuli, such as chemical changes of the ambient environment or light, may be enabled by using a similar system to that proposed herein, but with a different responsive functional material for the outer shell. ${ }^{[13]}$ This concept could potentially provide a wide range of applications ranging from tissue engineering $^{[14]}$ to macroscale self-assembly of materials. ${ }^{[15]}$

\section{Experimental Section}

PNIPAM coating: A thin layer of PNIPAM was deposited on hybrid Ge/parylene core/shell NW forests by photopolymerization of a solution mixture containing $N$-isopropylacrylamide (NIPAM) monomers, 2,2'-diethoxyacetophenone (DEAP) photoinitiator, and $N, N$ methylenebisacrylamide (bis-AMD) crosslinker. The solution mixture was prepared by mixing $1.6 \mathrm{mmol}$ of NIPAM, $30 \mu \mathrm{mol}$ of bisAMD, and $6 \mu \mathrm{mol}$ DEAP in $2 \mathrm{~mL}$ deionized water. The solution mixture was degassed with nitrogen gas for $10 \mathrm{~min}$ to remove dissolved oxygen in the solution. The resulting solution was spread on the NW arrays and squeezed by gently pressing a glass slide cover on top. Finally, the polymerization was performed by using a UV lamp $(365 \mathrm{~nm}, 15 \mathrm{~W})$ for $60 \mathrm{~min}$. These processing conditions resulted in a PNIPAM outer shell thickness of approximately $100 \mathrm{~nm}$ and the NW forests maintaining the nanofibrillar structures with some degree of aggregation, as evident from SEM analysis (Figure $1 \mathrm{~b}$ and c).

Macroscopic wet adhesion tests at different temperatures: The wet adhesion tests were performed by immersing the NW fasteners inside a PDMS liquid cell filled with water. Then, a normal preload stress was applied to engage the NW fasteners $\left(\mathrm{ca} .0 .5 \times 0.6 \mathrm{~cm}^{2}\right)$, then a shear force was applied, and finally the preload stress was removed to measure the pure shear adhesion strength. The shear stress at failure corresponds to the maximum shear adhesion strength. These adhesion tests were performed at different temperatures by checking 
the water temperature with using a thermometer immersed in the water bath on top of a hot plate (inset in Figure $3 \mathrm{a}$ ).

Received: August 25, 2009

Revised: October 21, 2009

Published online: December 8, 2009

Keywords: adhesion - gels - nanostructures - nanowires · noncovalent interactions

[1] C. Sanchez, H. Arribart, M. M. G. Guille, Nat. Mater. 2005, 4 277.

[2] a) K. Autumn, Y. A. Liang, S. T. Hsieh, W. Zesch, W.-P. Chan, W. T. Kenny, R. Fearing, R. J. Full, Nature 2000, 405, 681; b) A. K. Geim, S. V. Dubonos, I. V. Grigorieva, K. S. Novoselov, A. A. Zhukov, S. Yu. Shapoval, Nat. Mater. 2003, 2, 461; c) L. Ge, S. Sethi, L. Ci, P. M. Ajayan, A. Dhinojwala, Proc. Natl. Acad. Sci. USA 2007, 104, 10792; d) G. Huber, H. Mantz, R. Spolenak, K. Mecke, K. Jacobs, S. N. Gorb, E. Arzt, Proc. Natl. Acad. Sci. USA 2005, 102, 16293; e) L. Qu, L. Dai, M. Stone, Z. Xia, Z. L. Wang, Science 2008, 322, 238; f) M. Murphy, B. Aksak, M. Sitti, J. Adhes. Sci. Technol. 2007, 21, 1281.

[3] a) H. Ko, J. Lee, B. E. Schubert, Y.-L. Chueh, P. W. Leu, R. S Fearing, A. Javey, Nano Lett. 2009, 9, 2054; b) R. Kapadia, H.
Ko, Y.-L. Chueh, J. C. Ho, T. Takahashi, Z. Zhang, A. Javey, Appl. Phys. Lett. 2009, 94, 263110.

[4] a) H. G. Schild, Prog. Polym. Sci. 1992, 17, 163; b) L. Liang, X. Feng, J. Liu, P. C. Rieke, G. E. Fryxell, Macromolecules 1998, 31, 7845.

[5] D. L. Huber, R. P. Manginell, M. A. Samara, B. Kim, B. C. Bunker, Science 2003, 301, 352.

[6] M. Miwa, A. Nakajima, A. Fujishima, K. Hashimoto, T. Watanabe, Langmuir 2000, 16, 5754.

[7] H. Ko, Z. Zhang, Y.-L. Chueh, J. C. Ho, J. Lee, R. S. Fearing, A. Javey, Adv. Funct. Mater. 2009, 19, 3098.

[8] Intermolecular and Surface Forces; J. Israelachvili, Academic, New York, 1992.

[9] K. Iwai, K. Hanasaki, M. Yamamoto, J. Lumin. 2000, 87, 1289.

[10] J. Wu, B. Zhou, Z. Hu, Phys. Rev. Lett. 2003, 90, 048304.

[11] R. Yoshida, K. Uchida, Y. Kaneko, K. Sakal, A. Kikuchi, Y. Sakurai, T. Okano, Nature 1995, 374, 240.

[12] M. Shibayama, K. Nagai, Macromolecules 1999, 32, 7461.

[13] R. Yerushalmi, A. Scherz, M. E. van der Boom, H. B. Kraatz, J. Mater. Chem. 2005, 15, 4480 .

[14] S. Ohya, Y. Nakayama, T. Matsuda, Biomacromolecules 2001, 2, 856.

[15] G. Whitesides, M. Boncheva, Proc. Natl. Acad. Sci. USA 2002, $99,4769$. 


\section{Angewandte}

Supporting Information

(C) Wiley-VCH 2009

69451 Weinheim, Germany

Thermoresponsive Chemical Connectors Based on Hybrid Nanowire Forests**

Hyunhyub Ko, Zhenxing Zhang, Yu-Lun Chueh, Eduardo Saiz, and Ali Javey*

ange_200904724_sm_miscellaneous_information.pdf 


\section{Swelling/de-swelling of the thermoresponsive NW forests}

The swelling/de-swelling behavior of the thermoresponsive NW forests was studies by using a S4300SE/N field emission variable pressure SEM (Hitachi). After immersing the NW samples in a water bath at $\sim 25^{\circ} \mathrm{C}$ for $20 \mathrm{~min}$, the samples were taken out and immediately placed in the SEM chamber (100 Pa pressure in the chamber). The SEM images taken immediately after loading inside of the microscope correspond to that of the "wet" state while those taken $30 \mathrm{~min}$ after the vacuum pumping $\left(1 \times 10^{-3}\right.$ Pa pressure in the chamber) correspond to the dry state. It should be noted that the environmental SEM studies present only a rough approximation (lower bound limit) of the actual swelling/de-swelling of the NWs fasteners as the samples were not immersed in water during the imaging due to instrumentation limitation, in contrast to the performed adhesion experiments.

From environmental SEM studies (Fig. S1), we observed 6-10 \% change in the overall diameter of NWs $(750-850 \mathrm{~nm})$ when transitioning between the wet to dry states. Since the PNIPAM shell is $~ 100 \mathrm{~nm}$ thick, this corresponds to a thickness reduction of $25-40 \%$ for the PNIPAM outer shell, consistent with what is often reported in the literature for bulk samples prepared using nearly identical conditions. ${ }^{[1]}$ 

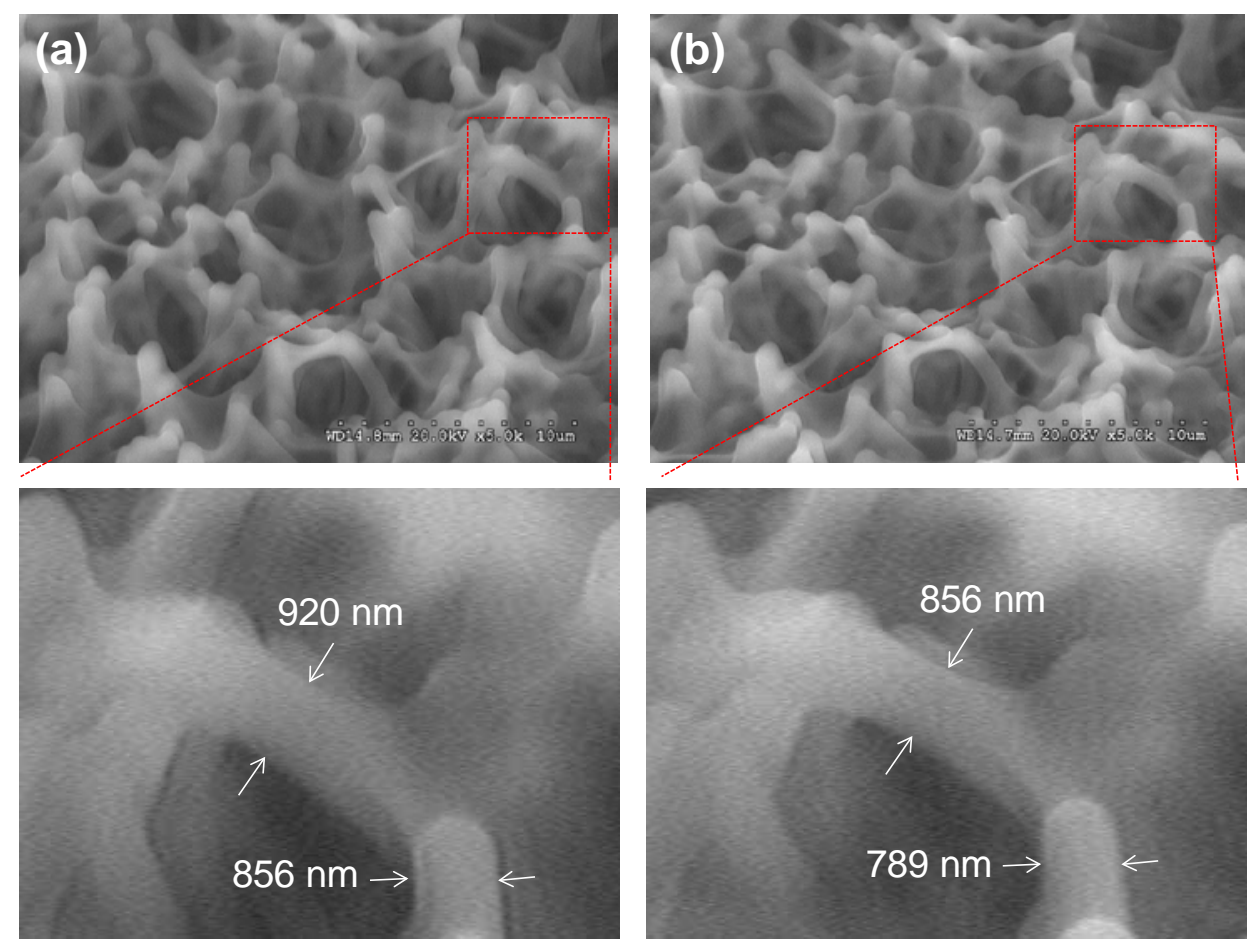

Figure S1. Top-view SEM images of PNIPAM/NW forests at (a) wet and (b) dry states. 


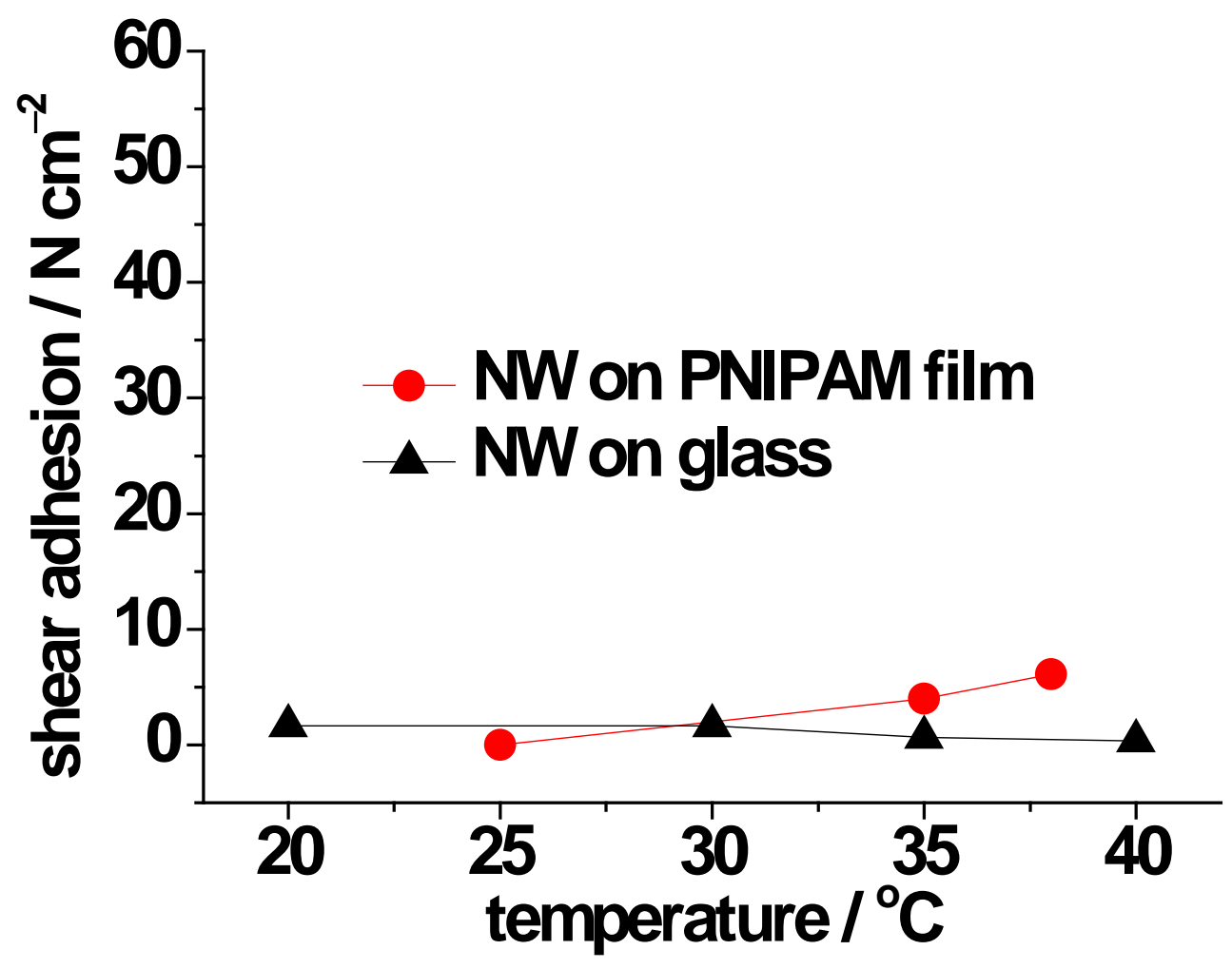

Figure S2. Shear wet adhesion strengths of the Ge/parylene/PNIPAM core/multishell NW forests on planar PNIPAM film (red curve) and planar glass substrate (black curve) at different water temperatures. 


\section{References}

[1] M. Shibayama, K. Nagai, Macromolecules 1999, 32, 7461. 